this solution gives, when proper conditions are established, the brick-red compound which might be considered as an intermediate product between the halide and the sulphide.

The three sulphohalides of lead are all readily prepared by this means. The bromosulphide is the most stable of the compounds and it can be prepared in a very striking manner by treating 2 to 3 grams of lead sulphide or galena with to to $\mathrm{I}_{5} \mathrm{cc}$. of strong hydrobromic acid, and diluting with a large volume of water. The acid should be allowed to act until hydrogen sulphide is freely evolved. The solution will retain sufficient gas so that when a liter of water is quicky added, the brick-red bromosulphide appears.

Hydrochloric and hydriodic acid solutions of galena, when freshly prepared and quickly diluted, behave in a similar manner. With the hydrochloric acid solution the conditions are better obtained when more acid is present than above noted for hydrobromic. When this solution is diluted with water, the bright red chlorosulphide appears, sometimes preceded by the white chloride. Excess of water invariably causes the chlorosulphide to change to the black lead sulphide, which is the final product. The formation of the bromosulphide by this method makes an extremely beautiful experiment; the simple addition of water to a freshly prepared solution of galena in hydrobromic acid gives bright yellow bromide which almost instantly changes to a bright red. On further diluting and allowing to stand, the black sulphide is obtained. These changes seem to indicate that the formation of these compounds is due to certain definite relations between the halide and hydrogen sulphide in a solution of the corresponding halogen acid.

UNIVERSITY OF WISCONSIN,

Madison, Wis.

\title{
A MODIFICATION OF THE SULPHURIC ACID TEST FOR FORIIALDEHYDE IN MILK.
}

By A. Gustay Luebert.

Receired July IO, zgor.

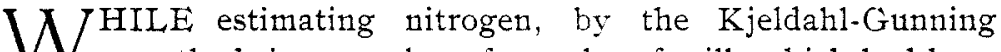
method, in a number of samples of milk which had been preserved by the addition of a small quantity of formaldehyde, a peculiar violet coloration of the potassium sulphate crystals and sulphuric acid, surrounding them, was noticed. Especially was 
this true when the milk was added to the potassium sulphate and the sulphuric acid carefully poured down the side of the digestion flask, so that the liquids did not immediately mix. The violet or reddish-violet color appeared only in those samples of milk which contained the formaldehyde; in all others caramelization ensued at once, the crystals and the liquid becoming first brown and then black.

A number of tests were made upon milk to which formaldehyde had been added, and in each case the preservative seemed to retard the oxidizing action of sulphuric acid, causing the violet coloration to appear and preventing the usual caramelization of the milk. In none of the samples where formaldehyde was added was a negative result obtained.

These trials led to the adoption of the following method for the detection of formaldehyde in samples of milk : 5 grams of coarsely powdered potassium sulphate are placed in a IoO cc. flask, $5 \mathrm{cc}$. of the suspected milk distributed over it by means of a pipette and ro $\mathrm{cc}$. of sulphuric acid (sp. gr. I.84), carefully poured down the side of the flask. It is now allowed to stand quietly until the color develops. If formaldehyde is present the violet coloration of the potassium sulphate takes place in a few minutes, the color gradually dispersing through the entire liquid. If no formaldehyde be present the liquid will at once assume a brown color, rapidly changing to black. Milk which has stood for several hours previous to testing gives the reaction even more rapidly than a fresh sample.

This test is sensitive to a dilution of at least one part formaldehyde in 250,000 parts of milk.

\section{NEW BOOKS.}

QUalitative ANalysis: A GUIDE IN QUalitative WORK, With Data FOR ANALYTICAL OPERATIONS AND LABORATORY METhods IN INORganic Chemistry. By Albert B. Prescotit and Otis C. Johnson. Fifth Revised and Enlarged Edition, Entirely Rewritter. New York : D. Van Nostrand Co. Igor. $x i+420$ pp. Price, $\$ 3.50$.

So much new matter has been added to this edition of Prescott and Johnson's manual that it is almost a new work. The subject matter has been enlarged over the former edition by fully onehalf, and the size of the book increased Ioo pages. The introduction of a section on "Solution and Ionization" will aid teachers in making the subject clear to beginners. Much descriptive 